\title{
Bending nearfield compensation in the context of vibroacoustic active control
}

\author{
M. Michau ${ }^{1, a}$, A. Berry ${ }^{2}$, P. Herzog ${ }^{3}$ And P. Micheau ${ }^{2}$ \\ 1 École Centrale de Marseille, 13013 Marseille, France \\ 2 Faculté de Génie Mécanique, GAUS, Université de Sherbrooke, Canada \\ ${ }^{3}$ Laboratoire de Mécanique et d'Acoustique, Marseille, France
}

Received 21 June 2013, Accepted 21 April 2014

\begin{abstract}
The efficiency of passive methods for noise reduction decreases at low frequency. In this frequency range active structural acoustic control (ASAC) can reduce sound radiation of structures by controlling their vibration usually using piezoelectric or electrodynamic transducers. Besides its global effect on the structure, a bending nearfield is observed, depending on the actuator type. In some ASAC strategies as in the virtual impedance approach, actuators and sensors are collocated. Although it does not affect the radiation of the structure, the local bending strongly modifies the transfer function between transducers. A method is proposed in order to identify and compensate it. The global part of the vibratory field is extracted by cancelling the contribution of higher order modes approximated as a stiffness. After compensation, the signal from the sensor is much more representative of the radiation and nearly independent of the nature and the size of the actuator.
\end{abstract}

Key words: Nearfield compensation / vibroacoustic active control

\section{Introduction}

Lightweight composite materials are widely used by the aerospace industry because they combine high stiffness and low mass. Unfortunately, they provide poor acoustical performance at low frequencies. Active structural acoustic control (ASAC) strategies allow for effective aircraft noise attenuation of low frequencies with control units integrated in the structure. The sound transmitted through the fuselage can thus be reduced by controlling the vibration of the interior skin of the sidewall (trim panel). By colocating an inertial actuator and an accelerometer, it is possible to control the force/velocity dual pair at one point of the panel [1]. It has been shown that, under pure bending assumption, piezoelectric devices can also form colocated, dual actuator-sensor pairs for active control of plate vibration [2]. Besides its strong physical sense, duality is an interesting property regarding control stability [3]. However, in such a colocated pair, the sensor is especially influenced by the nearfield effect of the actuator. In addition to the global behaviour of the structure, the excitation is the cause of a local bending, depending on the actuator type [4], that strongly modifies the transfer function between colocated transducers.

\footnotetext{
${ }^{a}$ Corresponding author:

marc.michau@centrale-marseille.fr
}

The radiation of a structure, driven by large scale deformations, is barely affected by its local deformation. It thus appears that the global part of vibratory field induced by the actuator is much more relevant to deal with the control problem. The compensation of the bending nearfield may therefore be necessary when developping strategies where the acoustic radiation is estimated from local vibration measurements [5]. This would avoid that the control attempts to cope with the nearfield strain not involved in the farfield radiation. By doing this, the dynamic range and robustness of iterative algorithms should be improved.

\section{Mechanical model}

In the following analysis, we consider only the effect of an actuator as an external source acting on the structure. The passive mechanical impedance of the actuator is thus neglected in order to simplify expressions. Two usual technologies are considered, which have different acting principle on the structure (Fig. 1). An inertial actuator (AI) induces an almost normal distribution of force density whereas a piezoceramic actuator (PZT) can be considered as applying bending moments to the structure. It was verified with analytical model and finite element 


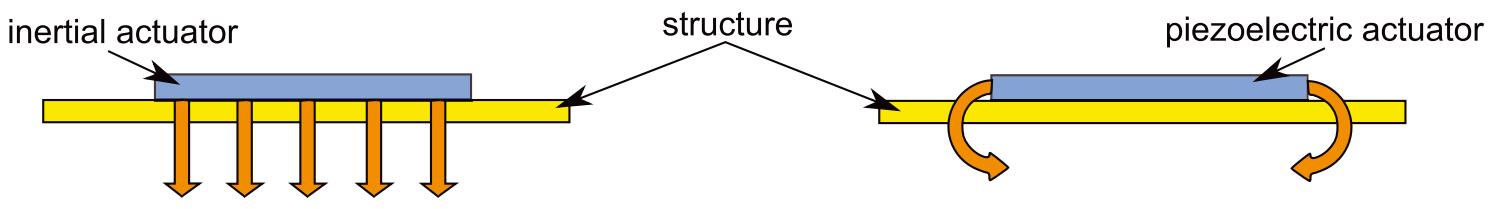

Fig. 1. Excitation mechanism for an inertial and a piezoelectric actuator on a structure.
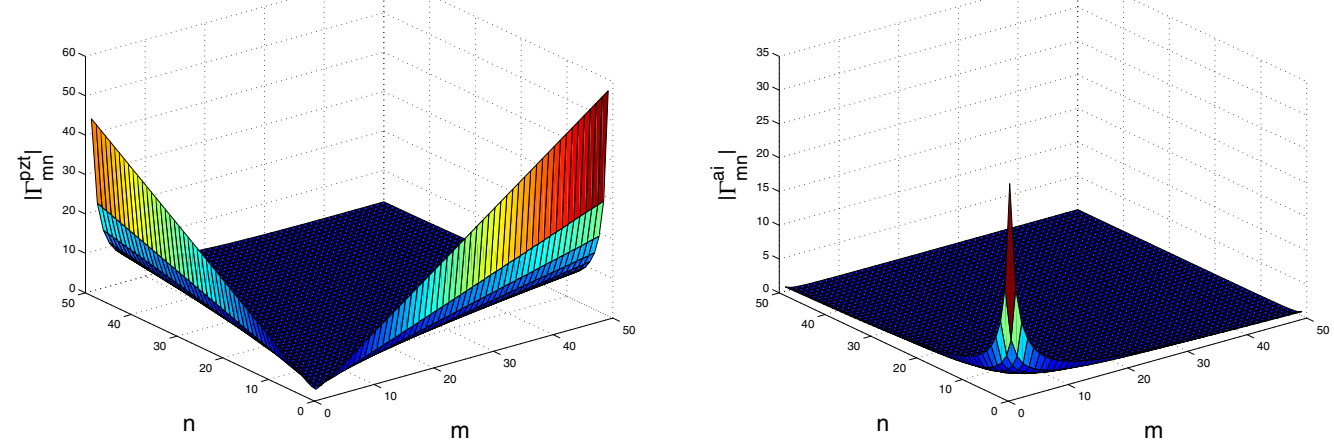

Fig. 2. Influence of the acting principle on the modal contribution for a piezoelectric (left) and an inertial (right) actuator.

method that the participation of the extension strain induced by the PZT to the transverse displacement of the structure was negligible. So, even with the same actuator size, the structure response may be quite different depending on which of these two technologies is involved.

The structure considered in this paper is a simply supported plate with dimensions $L_{x} \times L_{y} \times h$, a bending stiffness $D$ and a mass per unit area $M$. The excitation is harmonic with angular frequency $\omega$. Under pure bending assumption, an actuator-plate-sensor transfer is classically expanded over the structural modes:

$$
H(\omega)=\sum_{(m, n)} B_{m n} Y_{m n} C_{m n}
$$

where $Y_{m n}$ is the mechanical admittance of the $(m, n)$ mode:

$$
Y_{m n}=\frac{-j \omega}{M\left(\omega_{m n}^{2}-\omega^{2}\right)}
$$

with

$$
\begin{aligned}
\gamma_{m} & =\frac{m \pi}{L_{x}} \quad \text { and } \quad \gamma_{n}=\frac{n \pi}{L_{y}} \\
\omega_{m n} & =\sqrt{\frac{D}{M}}\left(\gamma_{m}^{2}+\gamma_{n}^{2}\right)
\end{aligned}
$$

$B_{m n}$ is a modal coupling coefficient beetween the actuator and the plate. It can be written:

$$
B_{m n}=\beta \Gamma_{m n} \Delta_{m n}^{a c t}
$$

where $\beta$ is a force per voltage constant. Expression (3) distinguishes the influence of the size and location from the actuator technology in the excitation mechanism. $\Delta_{m n}^{a c t}$ is dimensionless and depends on the actuator size and location. Defining $\left(x_{1}, x_{2}, y_{1}, y_{2}\right)$ its location, it is written as follows:

$$
\Delta_{m n}^{a c t}=\left[\cos \left(\gamma_{m} x_{2}\right)-\cos \left(\gamma_{m} x_{1}\right)\right]\left[\cos \left(\gamma_{n} y_{2}\right)-\cos \left(\gamma_{n} y_{1}\right)\right]
$$

Note that $\Delta_{m n}^{a c t}$ does not depend on the actuator technology. $\Gamma_{m n}$ is also a dimensionless modal coupling coeffcient, which expresses the actuator acting principle and is independant of its location:

$$
\Gamma_{m n}^{P Z T}=\left(\frac{\gamma_{m}^{2}+\gamma_{n}^{2}}{\gamma_{m} \gamma_{n}}\right) \quad \text { and } \quad \Gamma_{m n}^{A I}=\frac{1}{L_{x} L_{y}}\left(\frac{1}{\gamma_{m} \gamma_{n}}\right)
$$

Expression (5) may be interpreted as a series of contribution coefficients to the modal expansion. This is illustrated in Figure 2, where the coefficients have been calculated as a function of modal indices $(m, n)$. It appears that the piezoelectric actuator emphasizes the participation of higher order modes (small space scale), contrary to the inertial actuator. This difference leads to a different transverse displacement (Fig. 3). Unlike the inertial actuator, the piezoelectric actuator creates a strong bending nearfield effect. As $\Gamma_{m n}^{P Z T}$ increases with the modal index, it requires to consider much more terms in the expansion (1) with a piezoelectric than with an inertial actuator.

It should be noticed that the above expressions are theoretical and do not take into account some practical aspects. Especially, the piezoelectric device must be glued to the plate. This spreads the moments induced near its boundaries, leading to a reduced coupling with higher order modes.

The last component not yet considered in Equation (1) is the expression of the coupling coefficient $C_{m n}$ beetween the plate and the sensor. As far as dual pairs are considered, a piezoelectric polyvinylidene fluoride (PVDF) sensor is colocated to the piezoceramic actuator and an 

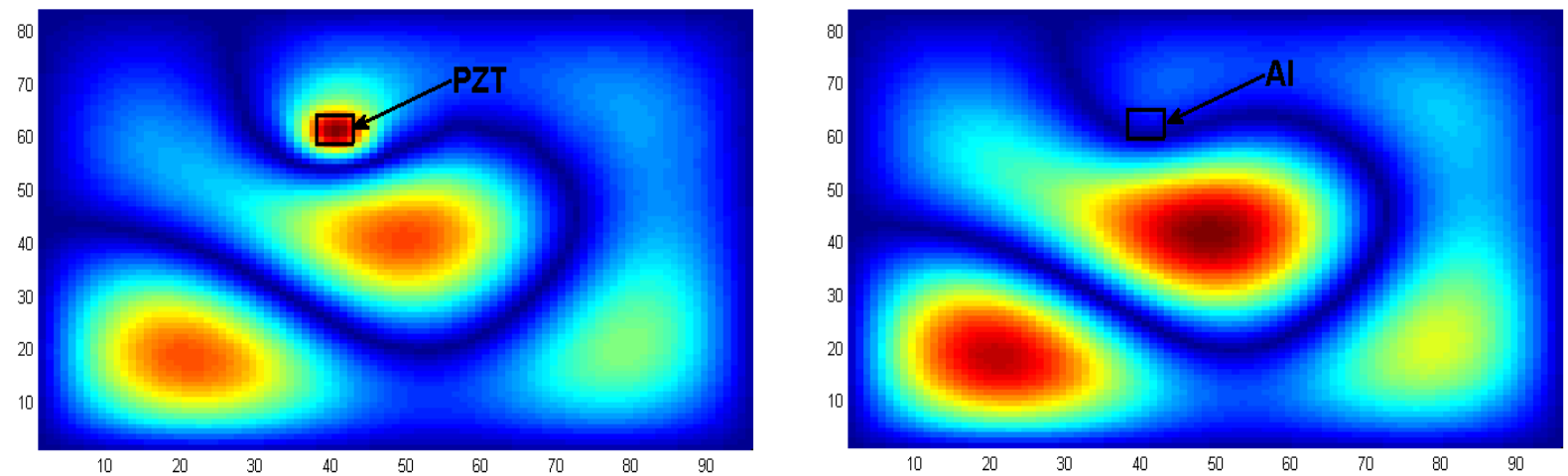

Fig. 3. Transverse displacement for a excitation by a piezoelectric and an inertial actuator, $f=400 \mathrm{~Hz}$.

accelerometer is colocated to the inertial actuator. Contrary to the PVDF sensor, the accelerometer is considered as a ponctual sensor:

$$
\Delta_{m n}^{A C C}=\sin \left(\gamma_{m} x_{a c c}\right) \sin \left(\gamma_{n} y_{a c c}\right) \neq \Delta_{m n}^{a c t}
$$

and

$$
C_{m n}^{A C C}=c^{A C C} \omega \sin \left(\gamma_{m} x_{a c c}\right) \sin \left(\gamma_{n} y_{a c c}\right)
$$

From the duality and the colocation it comes for the PVDF sensor:

$$
\Gamma_{m n}^{P V D F}=\Gamma_{m n}^{P Z T} \text { and } \Delta_{m n}^{P V D F}=\Delta_{m n}^{a c t}
$$

in

$$
C_{m n}^{P V D F}=c^{P V D F} \Gamma_{m n}^{P V D F} \Delta_{m n}^{P V D F}
$$

where both $c^{P V D F}$ and $c^{A C C}$ are voltage per velocity constants. It appears that the combined effect of $\Gamma_{m n}^{P V D F}$ and $\Gamma_{m n}^{P Z T}$ in the expression (1) still increase the difference in the number of terms to be considered depending on the technology chosen for the actuator/sensor pair.

\section{Nearfield compensation}

In the ASAC context, only the low frequency range is considered. The acoustical radiation to be controlled is mainly driven by the large scale deformation and is barely affected by the local strain described by higher order modes. For simulation purposes, a truncation of the modal expansion could be proposed, based on radiation criteria. Experimentally, the bending nearfield strongly modifies measured direct transfers. It then becomes difficult to estimate the structure radiation. A method is presented here in order to compensate this bending nearfield.

\subsection{Stiffness approximation of higher order modes}

The function $Y_{m n}: \omega \rightarrow \frac{-j \omega}{M\left(\omega_{m n}^{2}-\omega^{2}\right)}$ in $\mathcal{R}^{+}$is defined from (2). When the excitation frequency is much smaller than the natural frequency of a structural mode $\left(\frac{\omega^{2}}{\omega_{m n}^{2}} \ll 1\right), Y_{m n}$ is approched by its Taylor expansion in 0 at order 2 :

$$
Y_{m n}(\omega)=\frac{-j \omega}{M \omega_{m n}^{2}}+\circ\left(\omega^{3}\right)=\frac{-j \omega}{D\left(\gamma_{m}^{2}+\gamma_{n}^{2}\right)^{2}}+\circ\left(\omega^{3}\right)
$$

It can be shown that the participation of these modes can be expressed as a stiffness. Defining the two domains $E_{1}(\omega)$ the subset of modal indexes such as $\frac{\omega^{2}}{\omega_{m n}^{2}} \ll 1$ and $E_{2}(\omega)$ its complementary subset in $\mathcal{N}^{*} \times \mathcal{N}^{*}$ leads to the classical relations $[6]$ :

$$
\begin{aligned}
& Y_{m n}^{1}(\omega)=\frac{-j \omega}{M\left(\omega_{m n}^{2}\right)} \quad \forall(m, n) \in E_{1}(\omega) \\
& Y_{m n}^{2}(\omega)=\frac{-j \omega}{M\left(\omega_{m n}^{2}-\omega^{2}\right)} \quad \forall(m, n) \in E_{2}(\omega)
\end{aligned}
$$

The expression of $\frac{Y_{m n}^{1}}{j \omega}$ does not depend on $\omega$ but the repartition of the modes in the two domains $E_{1}$ and $E_{2}$ depends on the frequency and more specifically on the modal density. Note that the case $\left(\frac{\omega_{m n}^{2}}{\omega^{2}} \ll 1\right)$ could be treated as well in order to show that when the excitation frequency is much higher than the natural frequency of a mode, it can be approched by a mass. This is not considered in this paper.

\subsection{Theoretical results}

Considering the decomposition in two domains (9) and (10), it is then possible to express:

$$
H_{1}(\omega)=\sum_{(m, n) \in E_{1}(\omega)} B_{m n} Y_{m n}^{1} C_{m n}
$$

The compensated transfer function $\tilde{H}$ is defined as:

$$
\tilde{H}(\omega)=H(\omega)-H_{1}(\omega)
$$

By construction, $\tilde{H}$ respresents mainly the contribution of lower order modes, whatever the actuator technology considered. This is illustrated thereafter. When the excitation frequency is close to the natural frequency of a 


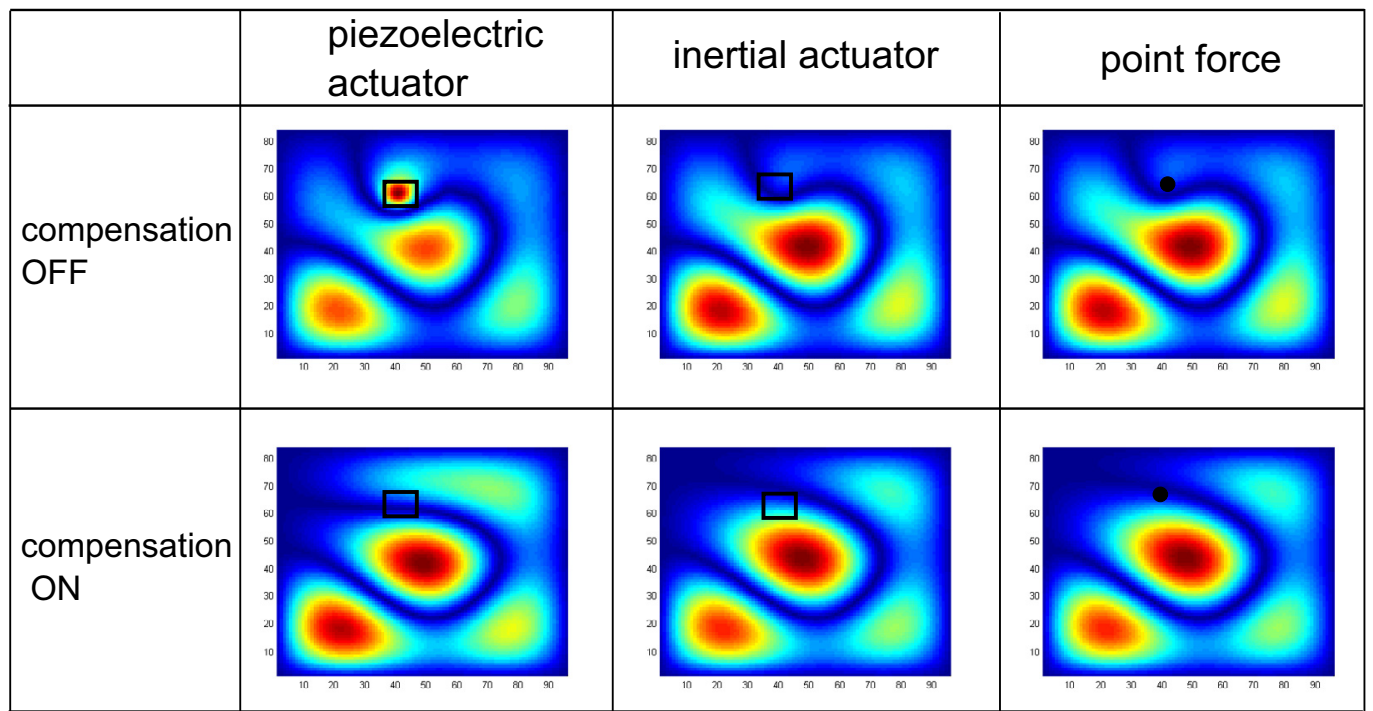

Fig. 4. Effect of the compensation on transverse displacement for different types of excitation, $f=400 \mathrm{~Hz}$.

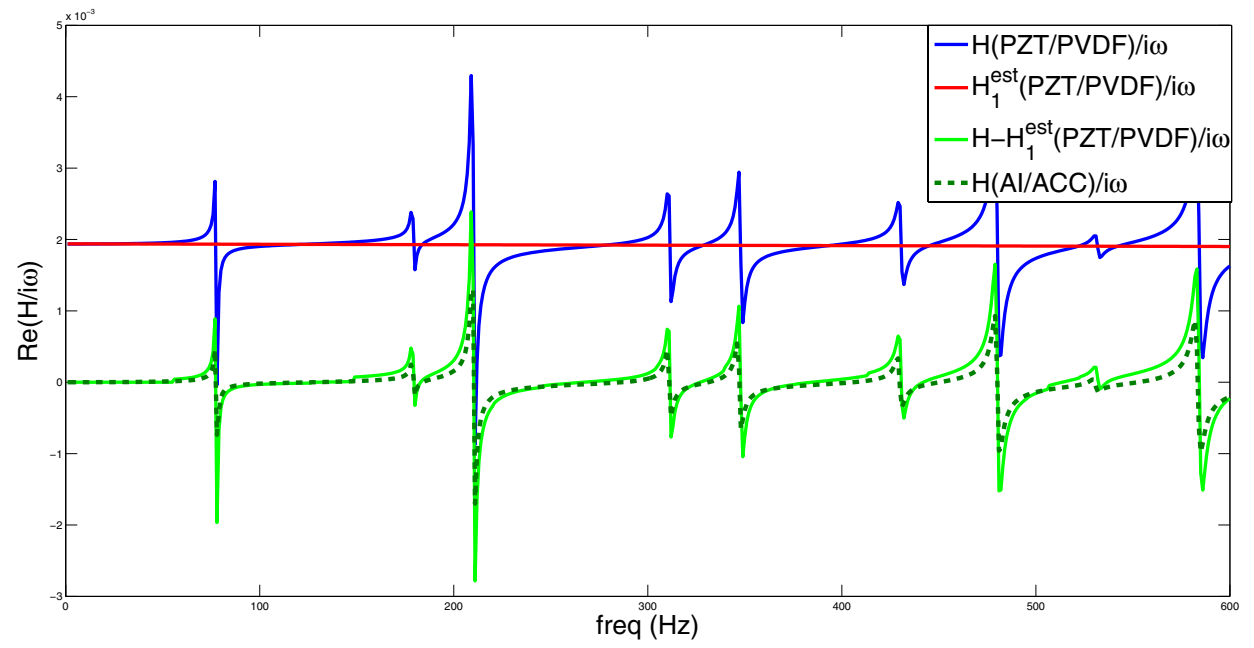

Fig. 5. Effect of the compensation on colocated dual actuator-sensor transfer.

mode, the transfer is dominated by this mode and neither the bending nearfield nor its compensation can be observed. Figure 4 corresponds to an off-resonance case $(f=400 \mathrm{~Hz})$. The effect of the compensation on the transverse displacement is presented for the two technologies previously considered, and for a point force excitation. The compensated transfers are much closer although the transverse displacement still depends on the excitation type. It can be explained by the difference in modal filtering $\left(\Gamma_{m n}^{p z t} \neq \Gamma_{m n}^{a i}\right.$ in Eq. (5)).

The compensation is then applied to the direct transfer beetween dual pairs (Fig. 5). By calculating $\frac{H}{j \omega}$, the higher order modes participation appears constant as calculated in Section 3.1. The compensation thus removes the contribution of higher order modes, which is almost constant at lower frequencies. The real part of $\frac{\tilde{H}}{j \omega}$ for the PZT has therefore an almost null mean value. For the inertial actuator/accelerometer pair, only the transfer without compensation is plotted. Neither the bending nearfield nor its compensation can be distinguished on the graph.

\subsection{Experimental procedure}

In practice, only the transfer $H(\omega)$ can be measured. As observed in the last section, $\frac{H_{1}}{j \omega}$ is almost constant above the $[10-600 \mathrm{~Hz}]$ frequency range. So a few offresonance measurements of $H(\omega)$ are enough in order to estimate $H_{1}$. Considering $H_{1}(\omega) \approx j \omega \tau$ where $\tau$ is a constant leads to a simple model in the time domain allowing to perform real-time compensation. This is a principle close to the method proposed in reference [7] in order to compensate the extensionnal modes participation. 


\section{Conclusions}

The method proposed here allows for a good distinction beetween global and local components of the vibration field in the case of colocated transducers. It has been shown that the bending nearfield strongly increases the number of terms in modal expansions to be considered in simulations. The repartition of modes in domains and the approximation of higher order modes realised in this study may help to fasten convergence of modal expansions. This paper also proposed a method to compensate the bending nearfield in order to perform a better estimation of global deformation. Such an information can be very usefull in the context of vibroacoustic active control. Because they have a different action mechanism, piezo and inertial actuators induce different deformation on the plate. The compensation presented here also allows to reduce the impact of these differences on the colocated control. The method presented here allows for estimating the bending nearfield compensation term. Its implementation with a feedback controller is an on-going work. A further step would be to find a configuration involving one or several piezoelectric devices of various form that induce the same global vibration to the structure as an inertial actuator, thus avoiding the need for such compensation.

\section{References}

[1] D. Guicking, J. Melcher, R. Wimmel, Active impedance control in mechanical structures, Acustica 69 (1989) 39-52

[2] Q. Sun, Some observations on physical duality and collocation of structural control sensors and actuators, J. Sound. Vib. 194 (1996) 765-770

[3] S.J. Elliott, P. Gardonio, T. Sors, M.J. Brennan, Active vibroacoustic control with multiple local feedback loops, J. Acoust. Soc. Am. 119 (2002) 1487-95

[4] M. Baudry, P. Micheau, A. Berry, Decentralized harmonic active vibration control of a flexible plate using piezoelectric actuator-sensor pairs, J. Acoust. Soc. Am. 125 (2009) 2978-86

[5] P. Masson, A. Berry, J. Nicolas, Active structural acoustic control using strain sensing, J. Acoust. Soc. Am. 102 (1997) 1588-99

[6] M. Géradin, D. Rixen, Mechanical vibrations: theory and application to structural dynamics, John Wiley, 1997, 425 p.

[7] Lee Young-Sup, P. Gardonio, S.J. Elliott, Coupling analysis of a matched piezoelectric sensor and actuator pair for vibration control of a smart beam, J. Acoust. Soc. Am. 111 (2002) 2715-26 